\title{
Simultaneous Multimodal Access to Wheelchair and Computer for People with Tetraplegia
}

\author{
Nazmus Sahadat \\ Georgia Institute of Technology \\ Atlanta, Georgia \\ m.n.sahadat@gatech.edu
}

\author{
Nordine Sebkhi \\ Georgia Institute of Technology \\ Atlanta, Georgia \\ sebnor31@gatech.edu
}

\author{
Maysam Ghovanloo \\ Georgia Institute of Technology \\ Atlanta, Georgia \\ mgh@gatech.edu
}

\begin{abstract}
Existing assistive technologies often capture and utilize a single remaining ability to assist people with tetraplegia which is unable to do complex interaction efficiently. In this work, we developed a multimodal assistive system (MAS) to utilize multiple remaining abilities (speech, tongue, and head motion) sequentially or simultaneously to facilitate complex computer interactions such as scrolling, drag and drop, and typing long sentences.

Inputs of MAS can be used to drive a wheelchair using only tongue motion, mouse functionalities (e.g., clicks, navigation) by combining the tongue and head motions. To enhance seamless interface, MAS processes both head and tongue motions in the headset with an average accuracy of $88.5 \%$.

In a pilot study, a modified center-out tapping task was performed by four able-bodied participants to navigate cursor, using head tracking, click using tongue command, and text entry through speech recognition, respectively. The average throughput in the final round was $1.28 \mathrm{bits} / \mathrm{s}$ and a cursor navigation path efficiency of $68.62 \%$.
\end{abstract}

\section{CCS CONCEPTS}

- Human-centered computing $\rightarrow$ Text input; Pointing; Gestural input; • Computer systems organization $\rightarrow$ Embedded hardware;

\section{KEYWORDS}

Tongue Motion, Head Tracking, Speech Recognition, Accessibility, Simultaneous Computer Interfaces, Human Interface Device.

\section{ACM Reference Format:}

Nazmus Sahadat, Nordine Sebkhi, and Maysam Ghovanloo. 2018. Simultaneous Multimodal Access to Wheelchair and Computer for People with Tetraplegia. In 2018 International Conference on Multimodal Interaction (ICMI '18), October 16-20, 2018, Boulder, CO, USA. ACM, New York, NY, USA, 7 pages. https://doi.org/10.1145/3242969.3242980

\section{INTRODUCTION}

Each year, 12,500 people with spinal cord injuries (SCI) are being added to an existing population of 276,000 in the United States

Permission to make digital or hard copies of all or part of this work for personal or classroom use is granted without fee provided that copies are not made or distributed for profit or commercial advantage and that copies bear this notice and the full citation on the first page. Copyrights for components of this work owned by others than ACM must be honored. Abstracting with credit is permitted. To copy otherwise, or republish, to post on servers or to redistribute to lists, requires prior specific permission and/or a fee. Request permissions from permissions@acm.org.

ICMI '18, October 16-20, 2018, Boulder, CO, USA

(c) 2018 Association for Computing Machinery.

ACM ISBN 978-1-4503-5692-3/18/10 . \$ \$15.00

https://doi.org/10.1145/3242969.3242980

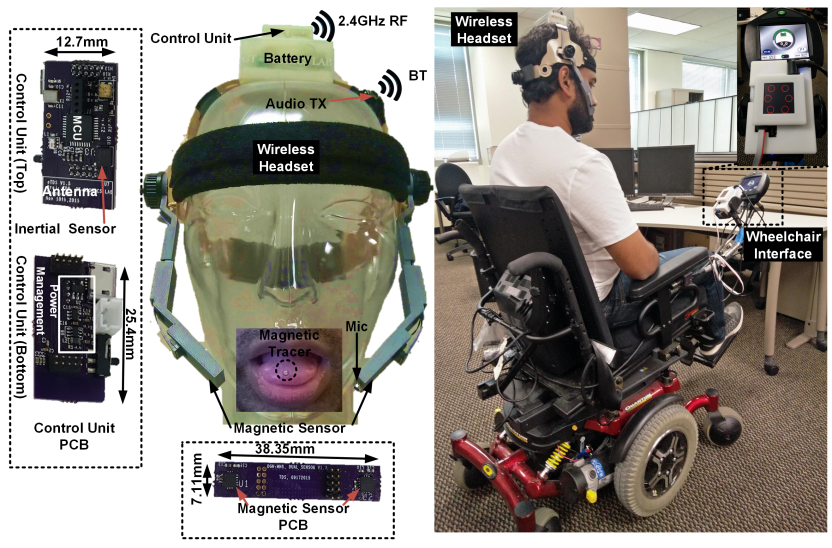

Figure 1: Multimodal Assistive System (MAS) during driving a wheelchair.

alone [20]. Among them, 92\% reside primarily in their home [21], and their age on average is only 29 years old. Currently, available treatments for people with tetraplegia are limited to regain their mobility to the degree that they can be fully autonomous again. They usually depend on caregivers and family members to do their daily life activities. This dependency not only impacts their lives but also the people around them. However, people with highlevel spinal cord injury (C1-C5) has remaining functional abilities on their neck up body parts (speech, eye, head, tongue, face, ear movements, and so forth). These remaining abilities can be used as inputs to control devices to provide them with more effective and efficient access to computers, wheelchairs, and smartphones. Some of the existing Assistive Technologies (ATs) leverage these remaining abilities by utilizing eye tracking [8], brain signal [9], tongue movement [3-6, 15, 16, 19], speech [17], and head movement [10]. A few limitations of previous ATs include good functionality only in specific environments (low noise, bright environment, flat terrain, and so forth), causing fatigue upon long usage, slow response, and being a constraint to a single modality with no redundancy. Tongue-controlled ATs are less affected by the surrounding environment and do not add muscle fatigue and has fast response time. However, complex tasks cannot be performed when using tongue as the sole modality. For instance, "drag and drop" requires multiple simultaneous inputs to select/drag/move/drop an icon to a specific location on a computer screen. Additionally, controlling the motion of a cursor with discrete commands is not efficient because it can only move in a vertical or horizontal direction. Typing sentences 
also take time with tongue-only ATs even with an efficient typing technique such as EdgeWrite [22], because multiple sequential commands are needed to type a single letter.

To overcome these obstacles, here we present a multimodal assistive system (MAS) in the form of an integrated wireless headset, shown in Figure 1, in which seven tongue gestures are translated into discrete commands, head motion is converted to continuous proportional input, and speech is recognized as text entry. To make MAS a compact, robust, and widely-used human interface device (HID) in future versions, head and tongue motions are captured and processed directly within the headset, in a built-in and ultra-low power control unit. Consequently, only the outputs that represent the user intentions (tongue commands, head orientation) are transmitted to target devices, such as PCs, smartphones, and power wheelchairs. The major contributions of this work are as follows:

- The first system, the size of a headset that can capture, process, and transmit commands from tongue and head motion, in addition to voice commands, to directly interface with smartphones, PCs, and power wheelchairs.

- A computationally efficient algorithm for detecting tongue commands from the raw magnetic field, generated by a tracer, entirely implemented in an embedded microcontroller with limited resources.

- Interface electronics that allow navigating a wheelchair using tongue commands.

- Emulating all computer mouse functions with a simultaneous combination of tongue commands and head motion.

- Evaluating performance and usability of the system by four able-bodied individuals, performing a modified center-out tapping task which includes typing.

\section{SYSTEM OVERVIEW}

Multimodal Assistive System (MAS) is a wireless headset composed of headgear with two 3D-printed sensor arms and a control unit, placed on top (Figure 1). Each sensor arm includes two 3axial magnetometers (LSM303D, STMicroelectronics) that capture changes in the magnetic field created by a tiny magnetic tracer attached near the tongue's tip. These magnetometers communicate with the control unit using a serial peripheral interface (SPI). Head motion is captured in the control unit by a 9-axis (LSM9DS1, STMicroelectronics) inertial measurement unit (IMU) composed of a 3-axial accelerometer, magnetometer, and gyroscope. Raw data from these sensors are sampled at $30 \mathrm{~Hz}$ and processed in a microcontroller unit (MCU) that includes a built-in proprietary $2.4 \mathrm{GHz}$ $\mathrm{RF}$ transceiver (CC2510, Texas Instruments) to transmit processed commands wirelessly to target devices (i.e., PC, smartphone, and wheelchair). Additionally, user's voice is captured by a microphone attached to the left arm of the headset and transmitted using a Bluetooth link. The average current consumption of this system is $21.8 \mathrm{~mA}$ which has 30 hours of battery lifetime using a $700 \mathrm{mAh}$ battery.

MAS interfaces with target devices using custom-designed components: a USB dongle for PCs and smartphones, and a modified BeagleBone Black for power wheelchairs which includes a wireless transceiver, oLED display, and driving logic circuits. A state machine is implemented in the receiver unit (Figure 1: wheelchair

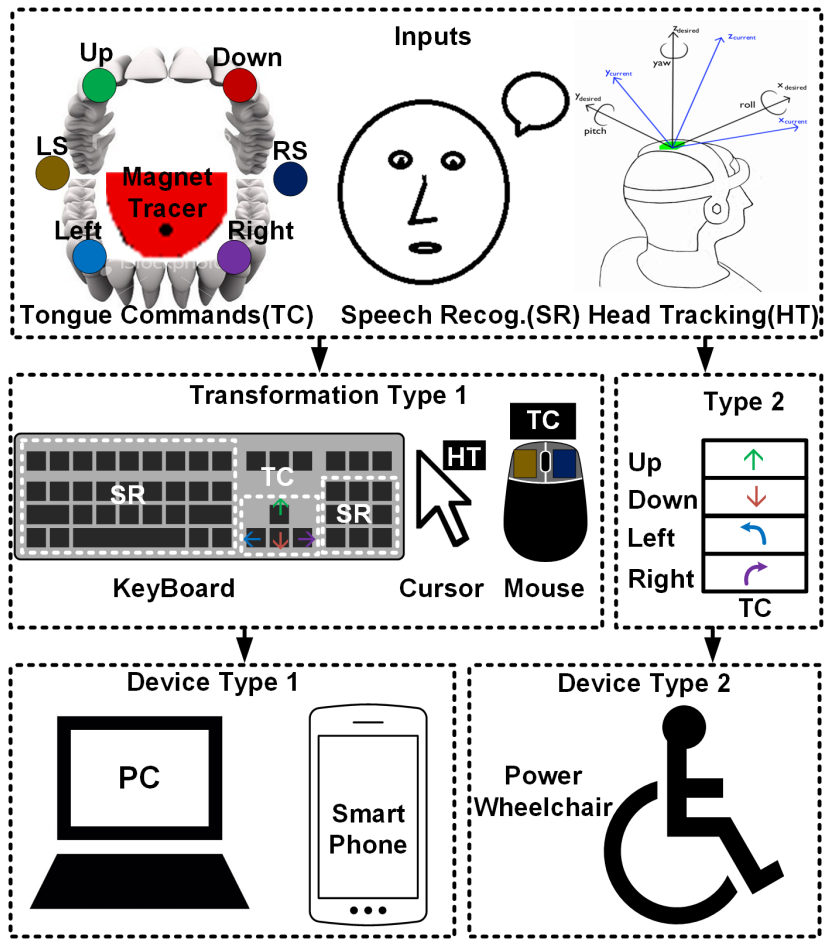

Figure 2: Three different inputs (Tongue commands, Head tracking and Speech recognition) are captured and transformed to different control strategies to interface with type 1 (PC, Smart phone) and type 2 (wheelchair) devices.

interface) which translates the assigned commands in different ways based on the device in use. For example, to interact with a PC, commands are translated into cursor movements ( $\mathrm{dx}, \mathrm{dy})$, arrow keys, and clicks as shown in Figure 2. Commands are translated to two driving vectors (forward/backward, left/right) and the receiver unit generates two analog voltages to drive a wheelchair, respectively.

\section{MODALITIES, GESTURES \& INTERFACES}

Depending on the type of application, the MAS user can select a single modality or use multiple modalities simultaneously or sequentially, as shown in Figure 2. Two out of seven tongue commands (LS and RS) are mapped to mouse buttons (left and right) in PCs and select/unselect in smartphones. Four tongue commands (up, down, left, right) are used for quick navigation between items (e.g., apps in smartphones or icons in PCs). Head motion controls the movement of a mouse cursor with $\mathrm{y}$ - and $\mathrm{x}$-axis positions determined by pitch and roll angles of the head, respectively. Cursor velocity is determined by a transformation model, described in Head Tracking subsection. A speech recognition software is used for text entry.

Driving a wheelchair requires modalities that generate commands that are surely intended by the user and remain unchanged and robust against disturbances from the surrounding environment, such as ambient noise, mechanical vibrations, inertia, and driving 
terrain. Among all of MAS modalities, voluntary tongue motion is the best candidate, since those commands are not affected by the acoustic noise, and robust against unintended body movements caused by driving through rough terrain. Indeed, in these situations, speech recognition and head tracking might not perform well and can generate unintended commands that might be dangerous to the driver. Considering these facts, four tongue commands (Left, Right, Up and Down) are used to drive the wheelchair through two different strategy: unlatched and latched modes. In the unlatched mode, the wheelchair stops when no commands are issued by the user. In the latched mode, forward or backward motion remains active (latched) after holding "Up" or "Down" command for more than a second, respectively. Consequently, the user is free to steer the wheelchair using the left or right command with angular speed being proportional to the dwelling time. Steering is deactivated when the tongue is returned to its resting position, and the wheelchair only moves in a straight line. The wheelchair can be brought to a standstill by issuing a "Down" command followed by the tongue returned to its resting position. The wheelchair can also be rotated left/right during latched mode independent of forward or backward commands.

\section{ALGORITHMS}

Figure 3 shows flowcharts of the tongue and head motion processing algorithms from the magnetometers and IMU data, respectively. Both of these algorithms run in the control unit's MCU.

\subsection{Tongue Commands Processing}

A calibration procedure ( 20 seconds) is required at the beginning of usage to estimate the gains and offsets of the four projection matrices that project the reference magnetometer's axes to each of the four magnetometers in the headset arms, using the least square error method in (1),

$$
\left[\begin{array}{ccc}
O_{x} & O_{y} & O_{z} \\
g_{x x} & g_{y x} & g_{z x} \\
g_{y x} & g_{y y} & g_{y x} \\
g_{z x} & g_{z y} & g_{z z}
\end{array}\right]=\left[1 \text { ref } s_{x, y, z}\right]^{-1} \times \operatorname{sens}_{x, y, z}
$$

where $\left(O_{x}, O_{y}, O_{z}\right)$ are the offsets, $\left(g_{x x}, g_{y y}, g_{z z}\right)$ are the gains, and $\left(g_{x y}, g_{x z}, g_{y x}, g_{y z}, g_{z x}, g_{z y}\right)$ are the cross gains, while $r e f s_{x, y, z}$ and sens $_{x, y, z}$ represent the reference and headset arms magnetometers' measurements, respectively.

To map tongue positions to commands, a short ( 70 seconds) training step is carried out to generate the 12-D (four 3-axial magnetometers) weight $\left(W_{21 \times 12}\right)$ and bias $\left(B_{21 \times 1}\right)$ matrices of the 21-linear binary support vector machines (SVM) (one vs. one, 7 commands), using the LIBSVM library [1].

To generate tongue commands, as shown in Figure 3 on the left, the first step is to attenuate ambient magnetic field interference, which is mainly the result of the earth's magnetic field (EMF). The EMF interference is captured by the reference sensor (9-D IMU's magnetometer), which is $10 \mathrm{~cm}$ away from the magnetic tracer not to be affected by it, and subtracted from the four magnetometers on the arms, using (2), (3), (4), and (5).

$$
\begin{aligned}
& \operatorname{sensprj}_{x}=r e f s_{x} \times g_{x x}+r e f s_{y} \times g_{x y}+r e f s_{z} \times g_{x z}+O_{x} \\
& \text { sensprj }_{y}=r e f s_{x} \times g_{y x}+r e f s_{y} \times g_{y y}+r e f s_{z} \times g_{y z}+O_{y}
\end{aligned}
$$

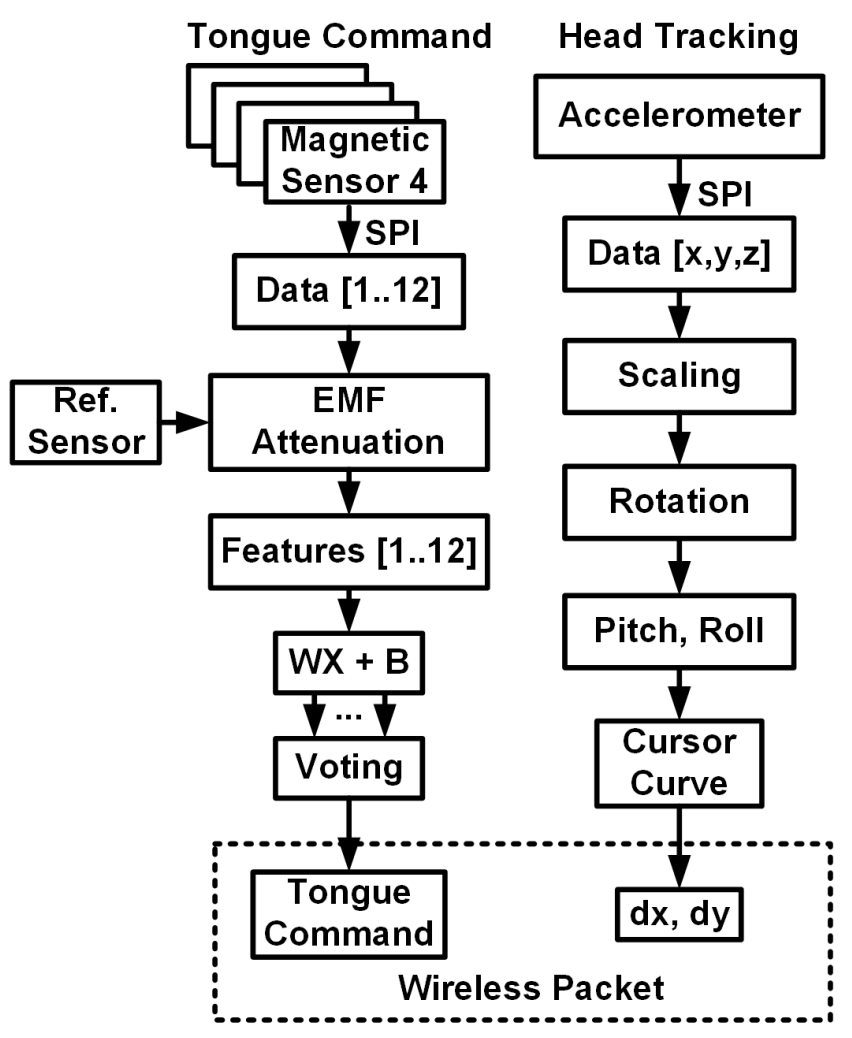

Figure 3: Flowcharts for generating tongue commands from magnetic field (left) and motion (right) vectors from the headset magnetometers and accelerometers, respectively.

$$
\begin{gathered}
\operatorname{sensprj}_{z}=\operatorname{refs}_{x} \times g_{z x}+\operatorname{ref} s_{y} \times g_{z y}+\operatorname{ref} s_{z} \times g_{z z}+O_{z} \\
\text { feature }_{x, y, z}=\operatorname{sens}_{x, y, z}-\operatorname{senspr} j_{x, y, z}
\end{gathered}
$$

where $\operatorname{sensprj}_{x, y, z}$ are the projected 3-axial reference sensor values and feature $x, y, z$ are 3-axial features generated from the four magnetometers on the arms after ambient magnetic field interference attenuation. Subsequently, the denoised 12-D features are classified using (6) and the maximum voted command $y$ is derived from the results, using (7) and saved in the wireless packet.

$$
\begin{aligned}
\text { res }_{21 \times 1} & =W_{21 \times 12} \times \text { feature }_{12 \times 1}-B_{21 \times 1} \\
y & =M A X(\operatorname{vote}(\text { res } 21 \times 1)),
\end{aligned}
$$

where $\operatorname{res}_{21 \times 1}$ is the 21 classification results.

\subsection{Head Motion Processing}

Head tracking algorithm, shown in Figure 3 on the right, processes the IMU's 3-D accelerometer data to generate mouse cursor movements in horizontal (dx) and vertical (dy) directions. Data is scaled using (8), then rotated to align the accelerometers' axes to the headset's frame of reference using (9),

$$
\begin{gathered}
\operatorname{accel}_{x, y, z}=\frac{\left(a_{x, y, z}-\text { offset }\right) \times \text { range }}{\text { scale }} \\
\operatorname{arot}_{x, y, z}={\text { Rotation } \times \text { accel }_{x, y, z}}^{\text {atal }}
\end{gathered}
$$




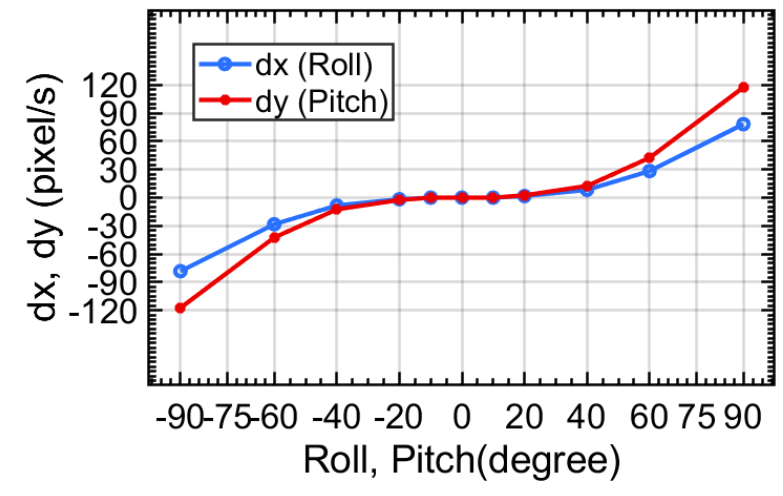

Figure 4: Mouse cursor movement model used to translate pitch, roll to horizontal and vertical direction.

where ( $a_{x, y, z}$, of $f$ set, range, scale, Rotation $)$ are the raw accelerometer $x, y$, and $z$ measurements, offset (32768), range $( \pm 2 g)$, scale (32768) and the rotation matrix to orient the sensor to the headset axis. Finally, accel $_{x, y, z}$ and $\operatorname{arot}_{x, y, z}$ are the adjusted accelerometer values after scaling and rotation. The rotation matrix is estimated in once-in-a-lifetime calibration. Accelerometer data is then used to find the pitch and roll of the head orientation using (10) and (11).

$$
\begin{gathered}
\text { Pitch }=\tan ^{-1}\left(\frac{-\operatorname{arot}_{x}}{\operatorname{arot}_{z}}\right) \times \frac{180^{\circ}}{\pi} \\
\text { Roll }=\tan ^{-1}\left(\frac{\operatorname{arot}_{y}}{\operatorname{arot}_{x}^{2}+\operatorname{arot}_{z}^{2}}\right) \times \frac{180^{\circ}}{\pi}
\end{gathered}
$$

Two 10-block piecewise linear models (Figure 4) are used to translate the roll and pitch $(-90,90)$ to horizontal $(\mathrm{dx})$ and vertical (dy) mouse cursor movement. A "no movement" zone is included to avoid undesired cursor movements for natural or small unintended head motion even at resting. The vertical cursor displacement gain is 1.5 times than the horizontal gain because of a limited range of motion in head pitch compared to roll.

\subsection{Speech Recognition}

User's voice is captured by a microphone and transmitted wirelessly using a separate Bluetooth transceiver. A commercial speech recognition software (Dragon Naturally Speaking, Nuance Communication) translates the audio data into text, which can be manipulated (e.g., copy, cut, paste, move) using the other features of the MAS.

\section{EVALUATION}

\subsection{Algorithm Accuracy}

The first evaluation focuses on the accuracy of the tongue command detection algorithm. Fifteen able-bodied participants were asked to position their tongue in the seven locations including resting (Figure 2) with four repetitions. Magnetometers' data were recorded for 10 seconds per command. Three repetitions were used to train the SVM model and one to assess the performance. Average true positive rate (TPR), false positive rate (FPR), and accuracy were calculated over the participants as metrics.

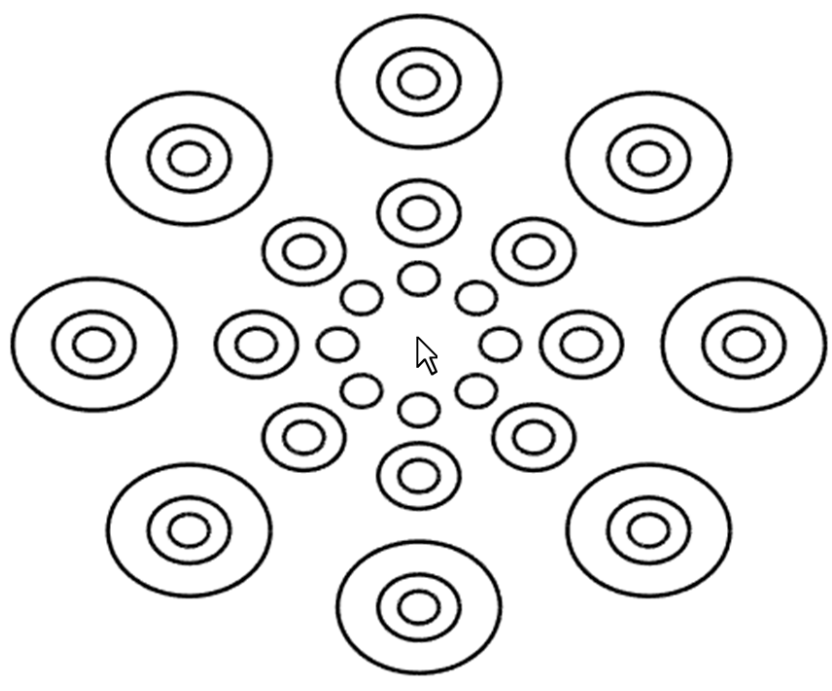

Figure 5: Targets in center-out tapping task with multiple dimensions based on index of difficulties.

Table 1: Center-out tapping task target diameter, W, distance, $\mathrm{D}$, and corresponding index of difficulties

\begin{tabular}{ccccc}
\hline Pixels & & D1 & D2 & D3 \\
\hline & & $\mathbf{6 1}$ & $\mathbf{1 2 2}$ & $\mathbf{2 4 4}$ \\
W1 & $\mathbf{3 0}$ & 1.60 & 2.34 & 3.19 \\
W2 & $\mathbf{6 1}$ & & 1.59 & 2.32 \\
W3 & $\mathbf{1 2 2}$ & & & 1.59 \\
\hline
\end{tabular}

\subsection{Pilot Study}

A preliminary usability study was also conducted where four ablebodied subjects were recruited to perform four repetitions of a center-out tapping task. The participant's tongue was dried with tissue and a small disk-shaped magnetic tracer $(\phi 4.8 \mathrm{~mm} \times 1.5 \mathrm{~mm}$, $\mathrm{K} \& \mathrm{~J}$ Magnetics, Jamison, PA), sterilized using isopropyl alcohol, was attached to the tongue at $1 \mathrm{~cm}$ from its tip using a cyanoacrylate tissue adhesive. Participants were asked to use head tracking to navigate the cursor and tongue command (Left Select) to select the target. Each repetition included 48 different targets whose size, distance, and the index of difficulty are reported in Table 1 and shown in Figure 5. Also, each repetition included two typing tasks that appeared in random order between the target selection. Participants were asked to type a sentence (1 4 words) using speech recognition, transfer the text to a specific text box and click on a button in the experiment's GUI using tongue commands and head motion.

The following parameters are used to assess the usability and performance of the system.

\subsection{Throughput (bits/s)}

Throughput $(T P)$ quantifies the information transfer rate between an input device to a computer. It is the ratio between the effective index of difficulty $\left(I D_{e}\right)$ to the average movement time $(M T)$ for a 
same D-W pair as shown in equation $(12)[2,18]$.

$$
T P=\frac{I D_{e}}{M T}
$$

Larger and closer targets are easier to reach and select compared to smaller and farther ones. $I D_{e}$ is obtained using Shannon's formula shown in (13).

$$
I D_{e}=\log _{2}\left(\frac{D_{e}}{W_{e}}+1\right)
$$

The effective distance, $D_{e}$, is the mean navigation distance traveled by a mouse cursor for all the same conditions. The effective width, $W_{e}$, is calculated using (14).

$$
W_{e}=4.133 \times S D_{x}
$$

$S D_{x}$ represents the standard deviation of the distance between the center of the target to the final cursor's position.

\subsection{Path Efficiency (\%)}

Path efficiency is the ratio between the minimum distance from the origin to the target divided by the actual distance traveled by the mouse pointer (15).

$$
\text { Path Efficiency }=\frac{\text { Min.nav.distance }}{\text { actualnavi.distance }} \times 100
$$

\subsection{Missed Target}

Each target reaching and selection time is limited to 20 seconds. If the participant is unable to reach and select a target within this time, the event is counted as a missed target.

\subsection{Typing Speed (wpm)}

Typing Speed is the ratio between the number of the words typed and task completion time (min). A "words typed" event is defined as typing the sentence using speech recognition, copy and transfer to a provided text box and click on a specific button in the experiment's GUI.

\subsection{Typing Accuracy (\%)}

Typing accuracy is the ratio between the total number of typed words as shown during the task to the total number of correct words typed by the participant.

\section{RESULTS}

\subsection{Algorithm Accuracy}

The average accuracy, the true positive ratio (\%) and the false positive ratio (\%) of tongue command's detection algorithm over 15 participants are shown in Table 2. The average seven tongue command detection accuracy varies from 82.43 to $88.50 \%$, TPR from 94.52 to $98.39 \%$ and FPR from 0.72 to $2.16 \%$. This algorithm has higher average TPR, lower FPR and requires less training time ( 70 seconds) than the algorithm used in [11].

\subsection{Pilot Study}

Throughput and path efficiency of the mouse cursor's navigation is shown in Figure 6. Average throughput varies over the trials from 1.06 to $1.28 \mathrm{bits} / \mathrm{second}$ and path efficiency from 66.67 to $69.08 \%$. Finally, the graph of movement time $(M T)$ to the index of difficulty
Table 2: Average Tongue command detection accuracy, true positive ratio and false positive ratio for 15 participants using a 4-fold validation technique for 7 commands.

\begin{tabular}{cccc}
\hline Trial No. & Accuracy & TPR (\%) & FPR (\%) \\
\hline T0 & 86.69 & 98.39 & 2.16 \\
T1 & 86.73 & 96.02 & 0.72 \\
T2 & 82.43 & 97.41 & 1.09 \\
T3 & 88.50 & 94.52 & 0.77 \\
\hline
\end{tabular}
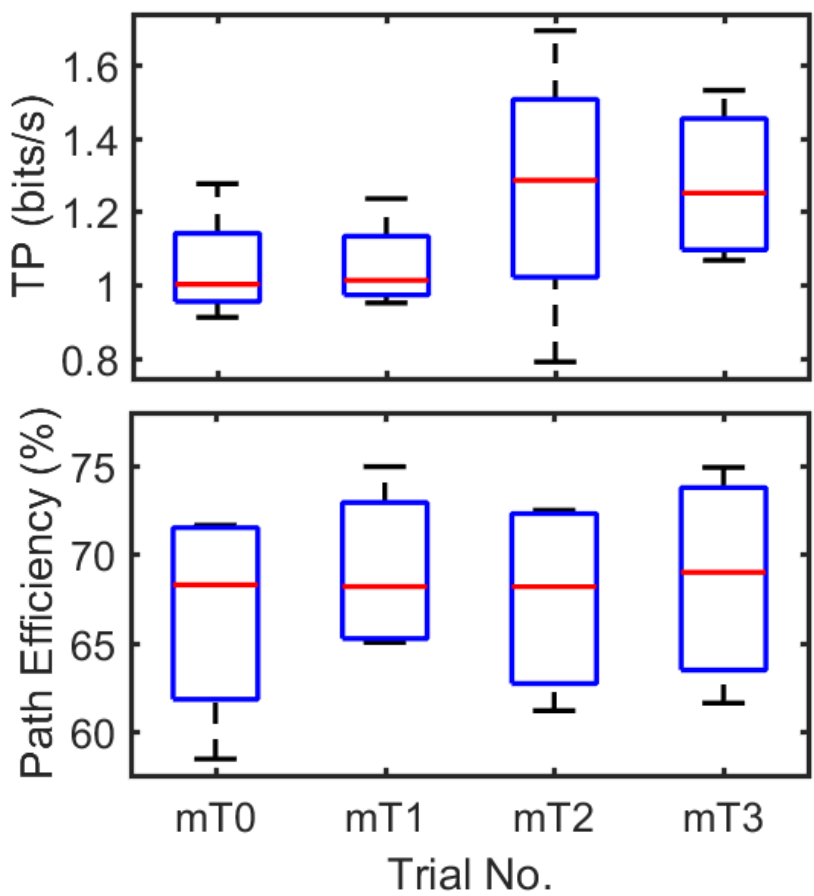

Figure 6: Throughput, TP (bits/s) and Path efficiency (\%) for center-out tapping task using all modalities.

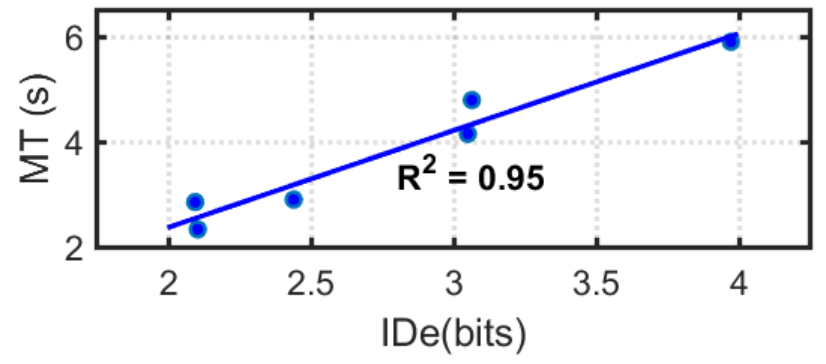

Figure 7: Movement time (MT) vs Index of difficulty (IDe) curve with a regression coefficient of 0.95 for repetition \#2.

$\left(I D_{e}\right)$ for repetition \#2 of all participants are plotted in Figure 7. $R^{2}$ for the linear fit of $M T$ vs. $I D_{e}$ is 0.95 which validates Fitts'law for this study. Average typing task completion time for all repetitions varies from 0.53 to 0.70 minute, average Typing task speed from 5.2 
Table 3: Benchmarking the Existing Tongue-Controlled Assistive Devices, TTK: Tongue Touch Keypad, TDS: Tongue Drive System, OSTG: optically sensing tongue gestures, ITCS: inductive tongue control system, MCU: microcontroller unit, RF: radio frequency, BT: Bluetooth, CU: central unit.

\begin{tabular}{cccccc}
\hline Features & TTK[6] & OSTG[16] & ITCS[19] & TDS[7] & This work \\
\hline Processor & MCU & PC & CU & PC/Phone & CC2510 \\
Standalone & yes & no & yes & no & yes \\
Connectivity & RF & RF & RF & RF & RF, BT, Wi-Fi \\
Proportional Input & no & no & yes(tongue) & no & yes( head tracking) \\
Visual Feedback & no & no & yes & no & yes \\
Obtrusive & no & no & no & yes & yes \\
\# of discrete commands & 9 & 4 & 18 & 7 & 7 \\
Command activation & Pressure & Optical & Inductive & Magnetic & Magnetic, Head orientation
\end{tabular}

to 6 wpm, average typing accuracy from 75 to $88.89 \%$, and average missed target from 5.73 to $10.42 \%$.

\section{DISCUSSION}

As demonstrated in the video figure, Multimodal Assistive System (MAS) can drive a power wheelchair, emulating a mouse and a keyboard to enable its user to perform complex PC tasks such as scrolling through a document, navigating through icons, playing a video game, among others. Although four able-bodied participants successfully evaluated MAS, it has yet to be assessed by its potential target population with tetraplegia. In future studies, the evaluation of the system will include different types of PC tasks such as maze navigation, playing a video game, sending an email and more challenging wheelchair driving in a constraint track.

A major limitation of the current system is a low data processing frequency of 30 commands/second. Further optimization of our data processing algorithms and implementation of a sensor fusion algorithm of all IMU data (accelerometer, gyroscope, and magnetometer) will provide a faster and more accurate mouse response [12-14]. To achieve these objectives, a hardware upgrade is needed where floating point computations can be done much faster than the current 8-bit MCU. We also plan to use a Bluetooth human interface device (HID) protocol to directly send integrated keyboard/mouse commands which will eliminate the USB receiver dongle to control computers or smartphones.

Among the three modalities, the control unit processes only the tongue and head motion information. It sends the audio signal directly to a smartphone / PC to be processed by the commercial speech recognition software. The tongue can be used either for speech or assigning a tongue command by moving the magnetic tracer. Therefore only two out of three inputs can be used simultaneously to control devices. However, speech recognition (SR) is only used to facilitate typing long sentences during a task like sending an email. The accuracy of SR is affected by the noisy environment and non-native English speaker accent. Since all participants in this study were non-native English speakers, this resulted in a low typing accuracy.

In this experiment, participants' typing time included typing a sentence (1 4 words) using speech recognition in a different dictation box then copy and transfer it to the experiment text box and click on a button. We observed that the majority of time was spent on transferring the typed sentence from the dictation box to the text box in the experiment's GUI. Including longer sentences with more words will significantly increase the typing task speed since the transfer and clicking time are constant.

Table 3 compares this work with the existing systems. MAS provides multiple wireless communication options, visual feedback, discrete and proportional inputs to control devices with in system processing capabilities to generate commands.

Only tongue commands are used in wheelchair driving. However, the other modalities can be used to perform auxiliary tasks such as driving mode switching between latched and unlatched. Since head tracking does not require calibration, it can also be used to trigger other modalities of the MAS by controlling the user interface, and training can be done by the users themselves. The MAS has the potential to help users (e.g., tetraplegics, ALS) to be more autonomous in accessing target devices (i.e., PCs, smartphones, power wheelchairs).

\section{CONCLUSION}

A novel multimodal assistive system (MAS) was presented in this work as a wearable system that can process both tongue and head motion in a built-in control unit, capture speech, and transmit commands wirelessly to control PCs, smartphones, and power wheelchairs. A usability testing was performed by four able-bodied participants in a modified center-out tapping task. Simultaneous multimodal inputs can provide people with tetraplegia efficient access to perform complex computer and smartphone tasks as well as drive their wheelchair comfortably.

\section{ACKNOWLEDGMENTS}

The authors would also like to thank the anonymous referees for their valuable comments and helpful suggestions.

This work was supported in part by the NSF awards CBET0828882 and IIS-0803184.

\section{REFERENCES}

[1] Chih-Chung Chang and Chih-Jen Lin. 2011. LIBSVM: a library for support vector machines. ACM transactions on intelligent systems and technology (TIST) 2, 3 (2011), 27.

[2] Paul M Fitts. 1954. The information capacity of the human motor system in controlling the amplitude of movement. Fournal of experimental psychology 47, 6 (1954), 381. 
[3] Maysam Ghovanloo, M Nazmus Sahadat, Zhenxuan Zhang, Fanpeng Kong, and Nordine Sebkhi. 2017. Tapping into tongue motion to substitute or augment upper limbs. In Micro-and Nanotechnology Sensors, Systems, and Applications IX Vol. 10194. International Society for Optics and Photonics, 1019413.

[4] Mayank Goel, Chen Zhao, Ruth Vinisha, and Shwetak N Patel. 2015. Tonguein-Cheek: Using Wireless Signals to Enable Non-Intrusive and Flexible Facial Gestures Detection. In Proceedings of the 33rd Annual ACM Conference on Human Factors in Computing Systems. ACM, 255-258.

[5] Xueliang Huo, Jia Wang, and Maysam Ghovanloo. 2008. Introduction and preliminary evaluation of the tongue drive system: Wireless tongue-operated assistive technology for people with little or no upper-limb function. Fournal of Rehabilitation Research \& Development 45, 6 (2008).

[6] Tongue Touch Keypad. [n. d.]. newAbilities Systems Inc. Santa Clara, CA available online www. newabilities. com ([n. d.]).

[7] Jeonghee Kim, Hangue Park, Joy Bruce, Erica Sutton, Diane Rowles, Deborah Pucci, Jaimee Holbrook, Julia Minocha, Beatrice Nardone, Dennis West, et al 2013. The tongue enables computer and wheelchair control for people with spinal cord injury. Science translational medicine 5, 213 (2013), 213ra166-213ra166.

[8] Aleksandra Królak and Paweł Strumiłło. 2012. Eye-blink detection system for human-computer interaction. Universal Access in the Information Society 11, 4 (2012), 409-419.

[9] Jinyi Long, Yuanqing Li, Hongtao Wang, Tianyou Yu, Jiahui Pan, and Feng Li. 2012. A hybrid brain computer interface to control the direction and speed of a simulated or real wheelchair. IEEE Transactions on Neural Systems and Rehabilitation Engineering 20, 5 (2012), 720-729.

[10] Ericka-Janet Rechy-Ramirez, Huosheng Hu, and Klaus McDonald-Maier. 2012 Head movements based control of an intelligent wheelchair in an indoor environment. In Robotics and Biomimetics (ROBIO), 2012 IEEE International Conference on. IEEE, 1464-1469.

[11] Elnaz Banan Sadeghian, Xueliang Huo, and Maysam Ghovanloo. 2011. Command detection and classification in tongue drive assistive technology. In Engineering in Medicine and Biology Society, EMBC, 2011 Annual International Conference of the IEEE. IEEE, 5465-5468.

[12] MN Sahadat, Arish Alreja, Pooja Srikrishnan, and Maysam Ghovanloo. 2015. A multimodal human computer interface combining head movement, speech and tongue motion for people with severe disabilities. In Biomedical Circuits and Systems Conference (BioCAS), 2015 IEEE. IEEE, 1-4.

[13] M Nazmus Sahadat, Arish Alreja, and Maysam Ghovanloo. 2018. Simultaneous multimodal PC access for people with disabilities by integrating head tracking, speech recognition, and tongue motion. IEEE transactions on biomedical circuits and systems 12, 1 (2018), 192-201.

[14] Nazmus Sahadat and Maysam Ghovanloo. 2017. Efficacy Assessment of multimodal Tongue Drive System (mTDS) in Comparison to Keyboard and Mouse (KnM). Archives of Physical Medicine and Rehabilitation 98, 12 (2017), e163-e164.

[15] Chris Salem and Shumin Zhai. 1997. An isometric tongue pointing device. In Proceedings of the ACM SIGCHI Conference on Human factors in computing systems. ACM, 538-539.

[16] T Scott Saponas, Daniel Kelly, Babak A Parviz, and Desney S Tan. 2009. Optically sensing tongue gestures for computer input. In Proceedings of the 22nd annual ACM symposium on User interface software and technology. ACM, 177-180.

[17] Andrej Škraba, Andrej Koložvari, Davorin Kofjač, and Radovan Stojanović. 2014. Prototype of speech controlled cloud based wheelchair platform for disabled persons. In Embedded Computing (MECO) 3rd Mediterranean Conference on. 162165.

[18] R William Soukoreff and I Scott MacKenzie. 2004. Towards a standard for pointing device evaluation, perspectives on 27 years of Fitts' law research in $\mathrm{HCI}$. International journal of human-computer studies 61, 6 (2004), 751-789.

[19] Lotte NS Andreasen Struijk. 2006. A tongue based control for disabled people. In International Conference on Computers for Handicapped Persons. Springer, 913 918.

[20] Non-Hispanic White and Non-Hispanic Black. 2016. Spinal Cord Injury (SCI) Facts and Figures at a Glance. (2016)

[21] Non-Hispanic White and Non-Hispanic Black. 2017. Spinal Cord Injury Facts \& Statistics. (2017). http://www.sci-info-pages.com/facts.htm

[22] Jacob O Wobbrock, Brad A Myers, and John A Kembel. 2003. EdgeWrite: a stylusbased text entry method designed for high accuracy and stability of motion. In Proceedings of the 16th annual ACM symposium on User interface software and technology. ACM, 61-70. 